\title{
Crossover from hydrodynamic to acoustic drag on quartz tuning forks in normal and superfluid ${ }^{4} \mathrm{He}$
}

\author{
D. I. Bradley, M. Človečko, S. N. Fisher, D. Garg, E. Guise, R. P. Haley, O. Kolosov, G. R. Pickett, and V. Tsepelin* \\ Physics Department, Lancaster University, Lancaster, LA1 4YB, United Kingdom \\ D. Schmoranzer and L. Skrbek \\ Faculty of Mathematics and Physics, Charles University, Ke Karlovu 3, 12116 Praha, Czech Republic \\ (Received 14 September 2011; revised manuscript received 5 December 2011; published 6 January 2012)
}

\begin{abstract}
We present measurements of the drag forces on quartz tuning forks oscillating at low velocities in normal and superfluid ${ }^{4} \mathrm{He}$. We have investigated the dissipative drag over a wide range of frequencies, from 6.5 to $600 \mathrm{kHz}$, by using arrays of forks with varying prong lengths and by exciting the forks in their fundamental and first overtone modes. At low frequencies the behavior is dominated by laminar hydrodynamic drag, governed by the fluid viscosity. At higher frequencies acoustic drag is dominant and is described well by a three-dimensional model of sound emission.
\end{abstract}

DOI: 10.1103/PhysRevB.85.014501

PACS number(s): 67.10.Jn, 43.38.+n, 47.37.+q

\section{INTRODUCTION}

Quartz tuning forks are piezoelectric mechanical oscillators with resonant frequencies typically in the kilohertz range. They are often used as frequency standards for timing devices such as digital watches. Since the two prongs of the fork oscillate in antiphase, the net force on the base is zero. This minimizes the dissipation and results in high intrinsic quality factors, $Q=f_{0} / \Delta f_{2}$, where $f_{0}$ is the resonance frequency and $\Delta f_{2}$ is the frequency width of the resonance. The $Q$ factors of quartz tuning forks are typically of order $10^{5}$ in vacuum at room temperature, and larger values can be obtained at very low temperatures.

Quartz tuning forks are also compact and easy to operate. They have found many applications in scientific research. For many years quartz tuning forks have been used in scanning probe microscopy. ${ }^{1-6}$ More recently they have been used in quantum fluids research to study various properties including viscosity, ${ }^{7,8}$ turbulence, ${ }^{9-15}$ cavitation, ${ }^{16,17}$ solubility, ${ }^{18,19}$ Andreev scattering, ${ }^{20-22}$ and coupling to acoustic modes. ${ }^{23,24}$

Here we present a detailed study of the drag forces on tuning forks oscillating in normal and superfluid ${ }^{4} \mathrm{He}$ over a wide frequency range. The measurements are made with custommanufactured arrays of quartz tuning forks. ${ }^{25}$ The forks have nominally identical geometries apart from the prong length, which varies along the array so that each fork has a different resonant frequency.

In Sec. II we describe the mechanics and equations governing the motion of a tuning fork. The electrical properties of quartz tuning forks are described in Sec. III, and our experimental arrangement is described in Sec. IV. Characterizations of the fork arrays in vacuum at $4.2 \mathrm{~K}$ are detailed in Sec. V. In Sec. VI we present detailed measurements at various temperatures, showing a clear crossover from hydrodynamic to acoustic-dominated drag on increasing frequency. We compare the measurements to the expected hydrodynamic drag forces and with models for the dissipative drag due to sound emission.

\section{EQUATIONS OF MOTION}

The motion of the tuning fork prongs can be described using Euler-Bernoulli beam theory. ${ }^{26}$ Each prong of the tuning fork is modeled as a beam of length $L$, thickness $T$, and width $W$, and the distance between the prongs is noted by $D$, as indicated in Fig. 1. The Euler-Bernoulli theory neglects the effects of shear deformation and is only applicable for thin beams $(T, W \ll L)$ and for small oscillation amplitudes. According to this model, the equation of motion for each prong can be written as

$$
\mu \frac{\partial^{2} u(x, t)}{\partial t^{2}}+\frac{\partial^{2}}{\partial x^{2}}\left(E I \frac{\partial^{2} u(x, t)}{\partial x^{2}}\right)=q(x, t),
$$

where $u(x, t)$ is the displacement of the prong at position $x$, $I=W T^{3} / 12$ is the area moment of inertia of the prong, $E=$ $7.87 \times 10^{10} \mathrm{~N} \mathrm{~m}^{-2}$ is Young's modulus for quartz, and $q(x, t)$ is the distributed load expressed in terms of a force per unit length. The mass per unit length is $\mu=\rho_{q} W T$, where $\rho_{q}=$ $2659 \mathrm{~kg} \mathrm{~m}^{-3}$ is the density of quartz. We model each prong as a cantilevered beam with one fixed end and one free end, giving rise to the following boundary conditions:

$$
\begin{array}{cc}
\text { Fixed end }:\left.u(x, t)\right|_{x=0}=0 & \left.\frac{\partial u(x, t)}{\partial x}\right|_{x=0}=0, \\
\text { Free end : }\left.\frac{\partial^{2} u(x, t)}{\partial x^{2}}\right|_{x=L}=0 & \left.\frac{\partial^{3} u(x, t)}{\partial x^{3}}\right|_{x=L}=0
\end{array}
$$

The equations above support free harmonic oscillations with resonant frequencies given by

$$
\omega_{n}=2 \pi f_{n}=\sqrt{\frac{E I}{\mu}} \frac{\beta_{n}^{2}}{L^{2}},
$$

where $\beta_{n}$ are solutions to

$$
\cos (\beta) \cosh (\beta)+1=0 .
$$

The solutions $\beta_{n}$ may be expressed in terms of the wave number $b_{n}$ of the $n$th mode of oscillation, $\beta_{n}=b_{n} L$. Equation (3) is solved numerically to yield the resonant frequencies: the fundamental mode has $\beta_{0}=1.87510$, and the first overtone mode has $\beta_{1}=4.69409$. $^{2,5}$

It is often useful to model the tuning fork as a simple harmonic oscillator with an effective mass $m_{\text {eff }}$ and a spring 
(a) Generator
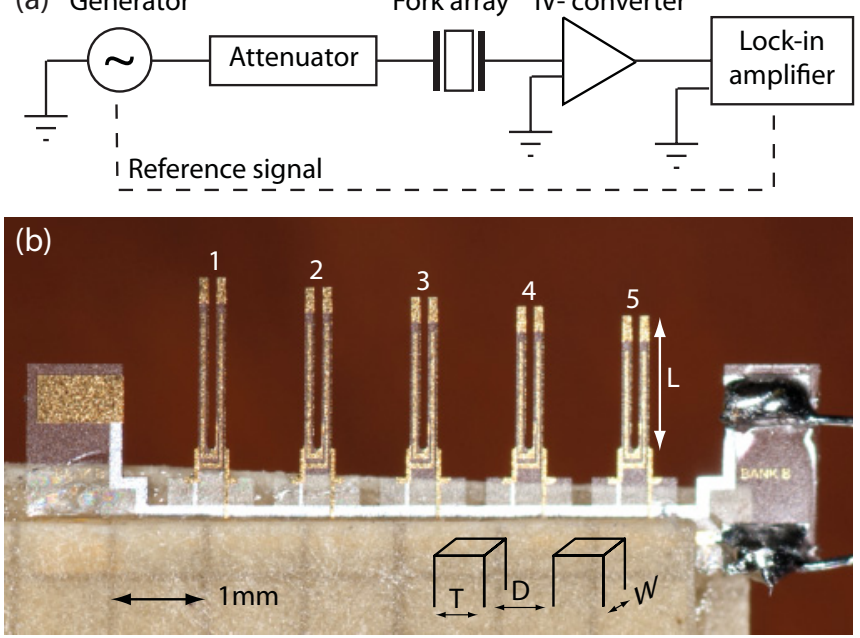

FIG. 1. (Color online) (a) Schematic of the electronic measurement scheme. (b) The custom-manufactured quartz tuning fork array. The base of the array is sandwiched between two sheets of Stycast-impregnated graph paper to mount it in the experimental cell (not shown).

constant $k_{n}$. The equation of motion for the tuning fork in the vicinity of a given resonance mode can thus be written as

$$
m_{\mathrm{eff}} \frac{d^{2} u(t)}{d t^{2}}+\gamma m_{\mathrm{eff}} \frac{d u(t)}{d t}+k_{n}^{2} u(t)=F,
$$

where $u(t)$ represents the displacement of the tip of each prong, $\gamma$ describes the drag force, and $F$ is the driving force. In a fluid, $\gamma$ has both real and imaginary components, $\gamma=i \gamma_{1}+\gamma_{2}$. The imaginary component $\gamma_{1}$ describes the nondissipative force arising from the fluid backflow around the tuning fork, while the real component $\gamma_{2}$ describes the dissipative drag force.

Using energetic considerations, ${ }^{26}$ the effective mass $m_{\text {eff }}^{(n)}$ and the spring constant $k_{n}$ of each resonant mode $n$ can be found from

$$
\begin{aligned}
\frac{1}{2} m_{\mathrm{eff}}^{(n)} v_{n}^{2} & =\frac{1}{2} \rho_{q} W T \int_{0}^{L}\left(\frac{\partial u_{n}(x)}{\partial t}\right)^{2} d x, \\
\frac{1}{2} k_{n} u_{n}^{2} & =\frac{1}{2} E I \int_{0}^{L}\left(\frac{\partial^{2} u_{n}(x)}{\partial x^{2}}\right)^{2} d x,
\end{aligned}
$$

where $u_{n}$ and $v_{n}$ are the displacement and velocity amplitudes of the prong tip, respectively, and $u_{n}(x)$ are the displacement amplitudes along the length of the prong. As shown in Appendix A, at resonance the effective mass is independent of the oscillation mode,

$$
m_{\mathrm{eff}}=\frac{1}{4} \rho_{q} W T L .
$$

The spring constant $k_{n}$ is found to be

$$
k_{n}=m_{\mathrm{eff}} \omega_{n}^{2}=\beta_{n}^{4} \frac{E I}{4 L^{3}} .
$$

So $k_{n}$ increases rapidly with increasing $n$. Away from resonance the situation is more complicated: for instance, for nonresonant frequencies where the tip amplitude is zero (i.e., where there is a node at the tip) the effective mass and spring constant will diverge.
In previous work w $^{2,8,10,13,15,24}$ the spring constant used is normally that defined with respect to static displacements of the prong tip, $k_{\text {stat }}=E W T^{3} / 4 L^{3}$, and the effective mass is then calculated as $m_{\text {eff }}^{(n)}=k_{\text {stat }} / \omega_{n}^{2}$ using Eq. (2). For the fundamental mode this approach yields $m_{\text {eff }}^{\text {stat }}=0.2427 \rho_{q} W T L$, which differs only by $3 \%$ from the value given by the dynamic theory [Eq. (7)]. However, we note that the static approximation will fail badly for the overtone modes. We further note that for the higher overtones the Euler-Bernoulli theory ceases to be valid, since shear deformations will become more and more important. In this case the Euler-Bernoulli model needs to be modified as given, for instance, by the theory of Timoshenko. ${ }^{26-28}$

A small alternating drive force, $F=F_{0} e^{-i \omega t}$, in the vicinity of a particular resonance leads to sinusoidal oscillations of the form $u(t)=u_{0} e^{-i \omega t}$. In this case, solving the equation of motion, Eq. (4), for the velocity amplitude of the tip of the prong, $v_{0}=i \omega u_{0}$, gives

$$
v_{0}=\frac{F_{0}}{m_{\mathrm{eff}}} \frac{\gamma_{2} \omega^{2}-i \omega\left(\omega_{n}^{2}-\omega^{2}-\omega \gamma_{1}\right)}{\left(\omega_{n}^{2}-\omega^{2}-\omega \gamma_{1}\right)^{2}+\gamma_{2}^{2} \omega^{2}},
$$

where $\omega_{n}^{2}=k_{n} / m_{\text {eff }}$ is the natural resonant frequency, and we assume that $\gamma_{1}$ and $\gamma_{2}$ are constants (i.e., the drag forces vary linearly with velocity). Here, the real and imaginary parts represent the components of the velocity which are in-phase and out-of-phase with the drive force, respectively. In practice the drag forces are usually very small $\left(\gamma_{1}, \gamma_{2} \ll \omega\right)$. In this case the resonance frequency is given by

$$
f_{0}=\frac{\omega_{n}}{2 \pi}-\frac{\gamma_{1}}{4 \pi}
$$

and the frequency width of the resonance is

$$
\Delta f_{2}=\frac{\gamma_{2}}{2 \pi} \text {. }
$$

At resonance, the velocity amplitude of the fork tip is maximized and is given by

$$
v_{0}^{\mathrm{res}}=\frac{F}{2 \pi m_{\mathrm{eff}} \Delta f_{2}} .
$$

\section{MEASUREMENTS OF QUARTZ TUNING FORKS}

To measure the resonant properties of a quartz tuning fork, an alternating voltage $V=V_{0} e^{-i \omega t}$ is applied to the electrodes on the surfaces of the prongs. This induces a piezoelectric force that sets the prongs into motion. The corresponding driving force on each prong is

$$
F=\frac{1}{2} a V,
$$

where $a$ is called the fork constant. A theoretical expression for the fork constant $a_{\mathrm{T}}$ can be obtained assuming ideal electrodes. For the fundamental mode, this is found to $\mathrm{be}^{5,8}$

$$
a_{\mathrm{T}}=3 d_{11} E \frac{T W}{L},
$$

where $d_{11}=2.31 \times 10^{-12} \mathrm{mV}^{-1}$ is the longitudinal piezoelectric modulus of quartz. The fork motion stresses the quartz and results in a piezoelectric current which is proportional to the tip velocity. The total power dissipated by the fork is $\dot{Q}=2\left(\frac{1}{2} F v\right)=\frac{1}{2} I V$ (the factor of 2 appears in the 
first equality since there are the two prongs), so the current must be given by ${ }^{5,8}$

$$
I=a v .
$$

An experimental value for the fork constant can be obtained from Eq. (12) by substituting Eqs. (13) and (15) for $F$ and $v$, respectively,

$$
a=\sqrt{\frac{4 \pi m_{\mathrm{eff}} \Delta f_{2} I_{0}^{\mathrm{res}}}{V}},
$$

where $I_{0}^{\text {res }}$ is the measured amplitude of signal current at the resonant frequency. With the exception of the effective mass, all of the quantities in Eq. (16) can be determined from the measurements. Measurements on various different quartz tuning forks ${ }^{5,8,29}$ show that the experimental value of the fork constant for the fundamental mode is about three times smaller than the theoretical value. The fork tip velocities inferred from the experimental fork constants are found to agree with optical measurements of the fork velocity to within about $10 \% .^{29}$

\section{EXPERIMENTAL}

A schematic of our measurement setup is illustrated in Fig. 1(a). The tuning fork was driven by an Agilent 33521 function generator, whose output was attenuated between 60 and $0 \mathrm{~dB}$. The current induced by the fork motion was converted to a voltage using a custom-made current-to-voltage converter $^{30}$ and the resulting voltage was measured using a lock-in amplifier. (We used a SR830 lock-in amplifier below $100 \mathrm{kHz}$ and a SR844 lock-in amplifier at higher frequencies.)

Figure 1(b) shows a custom-manufactured quartz tuning fork array. The arrays were mounted between two sheets of graph paper that were impregnated with Stycast 1266 for rigidity. For electrical connections a $75 \mu \mathrm{m}$ diameter copper wire was soldered to the contact pads. Each fork is mounted in parallel so all forks in the array are operated by the same pair of the leads. Due to the different prong lengths, the resonances of each fork are very well separated (see below), so the forks may be measured individually without any significant coupling between them.

All of the tuning forks had the same thickness, $W=75 \mu \mathrm{m}$, which was fixed by the wafer thickness. This was the minimum wafer thickness which could be used in the manufacturing process while maintaining a good success rate. (Around $90 \%$ of the arrays were fully operational.) There were 9 fork arrays and 30 individual tuning forks manufactured on each wafer. All of the forks also have the same prong thickness, $T=90 \mu \mathrm{m}$, and the same prong separation, $D=90 \mu \mathrm{m}$.

For characterization measurements at $4.2 \mathrm{~K}$, the forks were mounted in a vacuum chamber that was immersed in liquid helium in a transport dewar. Lower temperature measurements were carried out in a simple ${ }^{4} \mathrm{He}$ immersion cryostat with a temperature range of $1.55-4.2 \mathrm{~K}$. The temperature of the liquid helium was inferred from measurements of its saturated vapor pressure. $^{31}$

\section{TUNING FORK CHARACTERIZATION}

The tuning forks were characterized in vacuum at $4.2 \mathrm{~K}$. The resonant properties of each fork were measured by slowly
TABLE I. Results of the tuning fork characterization measure-

\begin{tabular}{|c|c|c|c|c|c|}
\hline Fork & $\begin{array}{c}L \\
(\mu \mathrm{m})\end{array}$ & $\begin{array}{c}f_{\mathrm{T}} \\
(\mathrm{kHz})\end{array}$ & $\begin{array}{c}f_{0} \\
(\mathrm{kHz})\end{array}$ & $\begin{array}{c}\mathrm{Q}_{\mathrm{vac}} \times 10^{-5} \\
\left(f_{0} / \Delta f_{2}\right)\end{array}$ & $\begin{array}{l}a \times 10^{7} \\
\left(\mathrm{NV}^{-1}\right)\end{array}$ \\
\hline W3S1 & 3500 & 6.457 & 6.757 & 4.6 & 3.93 \\
\hline fov & & 40.464 & 42.195 & 2.9 & 14.5 \\
\hline W3S4 & 2200 & 16.342 & 16.983 & 3.5 & 5.86 \\
\hline fov & & 102.414 & 105.618 & 0.31 & 22.6 \\
\hline W3L1 & 1900 & 21.910 & 22.502 & 9.4 & 6.77 \\
\hline fov & & 137.308 & 139.383 & 0.32 & 24.2 \\
\hline W3L2 & 1500 & 35.153 & 35.905 & 0.13 & 7.84 \\
\hline fov & & 220.303 & 220.948 & 0.13 & 29.7 \\
\hline W3L3 & 1200 & 54.927 & 55.555 & 5.0 & 9.45 \\
\hline fov & & 344.224 & 338.990 & 0.61 & 35.3 \\
\hline W3L4 & 900 & 97.648 & 97.694 & 1.4 & 11.9 \\
\hline fov & & 611.953 & 581.861 & 0.04 & 46.6 \\
\hline W3L5 & 700 & 161.418 & 159.361 & 2.8 & 14.8 \\
\hline fov & & 1011.600 & 920.271 & 0.13 & 58.3 \\
\hline W5H1 & 1888 & 22.189 & 23.020 & 2.9 & 7.01 \\
\hline fov & & 139.059 & 142.243 & 5.1 & 25.3 \\
\hline W5H2 & 1788 & 24.741 & 25.536 & 2.6 & 6.96 \\
\hline fov & & 155.049 & 157.812 & 3.9 & 27.5 \\
\hline W5H3 & 1688 & 27.759 & 28.562 & 5.4 & 7.66 \\
\hline fov & & 173.964 & 176.383 & 5.4 & 29.2 \\
\hline W5H4 & 1588 & 31.365 & 32.250 & 2.5 & 7.85 \\
\hline fov & & 196.563 & 198.785 & 2.3 & 30.4 \\
\hline W5H5 & 1488 & 35.723 & 36.727 & 6.4 & 8.41 \\
\hline fov & & 223.871 & 225.967 & 4.4 & 32.8 \\
\hline
\end{tabular}
ments at $4.2 \mathrm{~K}$ in vacuum (see text).

sweeping the excitation frequency through the resonance at various low-excitation amplitudes while measuring the resulting signals. (Both the in-phase and out-of-phase signals were measured using the lock-in amplifier.) The resonances were found to fit well to the ideal Lorentzian line-shape, given by Eq. (9), from which we can extract the resonant properties. The results are presented in Table I.

The first column of Table I labels the individual forks: W3 and $\mathrm{W} 5$ refer to two different quartz wafers; $\mathrm{L}$ and $\mathrm{H}$ refer to different arrays; $\mathrm{S}$ refers to single forks; and the number labels the particular tuning fork. The prong length of each tuning fork is shown in the second column. The third column shows the theoretical resonance frequencies $f_{\mathrm{T}}$ calculated using Eq. (2). The measured resonant frequency $f_{0}$ is listed in the fourth column of the table. For each fork the properties of the fundamental mode are listed in the first row, and those for the first overtone mode are listed in the second row. The theoretical resonance frequencies for the fundamental mode differ from the experimental ones by less than $4.5 \%$. The discrepancies between the resonant frequencies are probably accounted for by uncertainties and imperfections in the geometry. Also, we note that the theoretical values do not account for the presence of the electrodes. The larger discrepancies observed for the higher frequency, first overtone modes may indicate that rotary inertia is becoming important in this case. ${ }^{32}$

The quality factor for each fork resonance is listed in the fifth column. In general our measurements suggest that the quality factor of the forks decreases as the frequency increases; however, for some forks the quality factor for the first overtone is comparable to that of the fundamental mode. We note that 


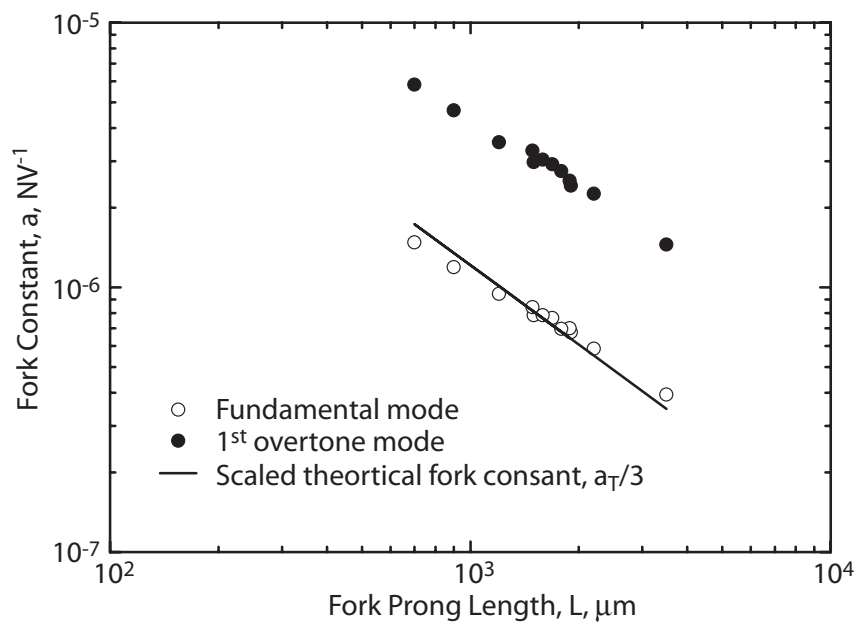

FIG. 2. The experimental values of the fork constant of the fundamental and first overtone modes as a function of the fork prong length $L$. The solid line corresponds to one third of the theoretical fork constant, $a_{T} / 3$, for the fundamental mode according to Eq. (14).

the mounting of the base of the forks/arrays can affect the quality factors: in some earlier measurements, we found that the quality factors were significantly lower when the base was not firmly anchored.

The last column of Table I lists the measured values for the tuning fork constant determined from Eq. (16) using the effective mass given by Eq. (7). The experimental values for both resonance modes are plotted as a function of the fork prong length in Fig. 2. The ratio between the measured and theoretical fork constants is approximately one third for the fundamental mode, as shown by the solid line in the figure. This agrees well with earlier measurements. $5,8,29$

The measured fork constant of the first overtone mode is nearly four times larger than that of the fundamental mode. We are not aware of any theoretical estimates of the fork constant for the first overtone mode. We further note that the surface electrodes of our forks are optimized for measurements of the fundamental mode, so it is likely that they will only collect a fraction of the induced charge in the first overtone mode.

\section{MEASUREMENTS IN LIQUID HELIUM}

Measurements of the resonant frequency and of the frequency widths (damping) of the fork resonances were made in liquid ${ }^{4} \mathrm{He}$ at various temperatures between 4.2 and $1.55 \mathrm{~K}$. Figure 3 shows the frequency width (damping) of the fork resonances as a function of the resonant frequency both in normal ${ }^{4} \mathrm{He}$ at $4.2 \mathrm{~K}$ and in superfluid ${ }^{4} \mathrm{He}$ at $1.55 \mathrm{~K}$. The small contribution from the intrinsic damping has been removed by subtracting the frequency width measured in vacuum at $4.2 \mathrm{~K}$.

The measurements in Fig. 3 reveal two very different behaviors depending on the frequency. In the low-frequency regime, the damping increases slowly with increasing frequency, while at higher frequencies there is a much steeper increase. We attribute this behavior to the crossover between hydrodynamic-dominated damping at low frequencies and acoustic-dominated damping at higher frequencies. The fitted

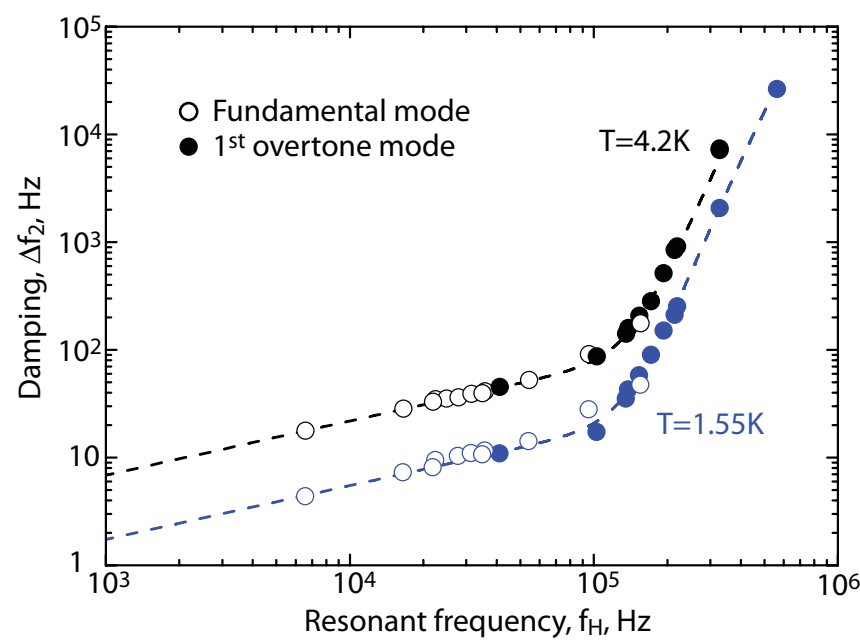

FIG. 3. (Color online) The frequency width (damping) of the fork resonances in normal ${ }^{4} \mathrm{He}$ at $4.2 \mathrm{~K}$ (black points) and in superfluid helium ${ }^{4} \mathrm{He}$ at $1.55 \mathrm{~K}$ (blue points) as a function of the resonant frequency. The intrinsic damping contribution measured in vacuum at $4.2 \mathrm{~K}$ has been subtracted. Open and closed symbols correspond to measurements of fundamental and first overtone modes, respectively. The dashed lines show the sum of the hydrodynamic damping and the damping according to a three-dimensional model for longitudinal quadrupole emission of sound (see text).

lines in Fig. 3 are described further below. We note that acoustic emission of tuning forks in liquid helium has been observed previously. ${ }^{23,24}$

In subsection A below, we describe the hydrodynamic drag forces measured at the lower frequencies and compare them with expected values based on the known properties of liquid helium. In subsection B we describe the acoustic contribution to the dissipative drag and compare our measured values with model calculations.

\section{A. Hydrodynamic drag}

When immersed in a fluid, the resonant frequency and the frequency width of the fork resonances are affected by the hydrodynamic drag forces acting on the oscillating prongs. The decrease in the resonant frequency can be described in terms of a mass enhancement of the oscillating object which has two contributions: fluid backflow around the moving prongs and viscous drag. The effective mass of the fork prongs in liquid helium can thus be written as the sum of three terms:

$$
m_{\mathrm{He}}=m_{\text {eff }}+\beta \rho_{\mathrm{H}} V+B \rho_{\mathrm{nf}} S \delta .
$$

The first term, $m_{\text {eff }}$, is the effective mass in vacuum, given by Eq. (7). The second term is the fluid backflow where $V=$ $T W L$ is the volume of each prong, $\rho_{\mathrm{H}}$ is the density of helium, and $\beta$ is a geometrical factor of order of unity. The third term arises from the viscous drag of the normal fluid, where $\rho_{\mathrm{nf}}$ is the normal fluid density of He-II (or the total fluid density above the superfluid transition temperature), $S=2 L T+2 L W$ is the surface area of each prong, and $B$ is a further geometrical factor of order of unity. The viscous penetration depth $\delta$ is given by

$$
\delta=\sqrt{\frac{\eta}{\pi \rho_{\mathrm{nf}} f}},
$$


TABLE II. Fitting parameters for the resonant properties measured in liquid ${ }^{4} \mathrm{He}$, assuming only hydrodynamic drag. The fits are very good for frequencies which are well below $100 \mathrm{kHz}$, but they fail at higher frequencies, indicating the onset of additional drag.

\begin{tabular}{|c|c|c|c|c|c|}
\hline Fork & $\begin{array}{c}f_{0} \\
(\mathrm{kHz})\end{array}$ & $\begin{array}{c}L \\
(\mu \mathrm{m})\end{array}$ & $\beta$ & $B$ & C \\
\hline $\begin{array}{c}\text { W3S1 } \\
\text { fov }\end{array}$ & $\begin{array}{r}6.757 \\
42.195\end{array}$ & 3500 & $\begin{array}{l}0.265 \\
0.265\end{array}$ & $\begin{array}{l}0.253 \\
0.250\end{array}$ & $\begin{array}{l}0.552 \\
0.570\end{array}$ \\
\hline W3L1 & 22.502 & 1900 & 0.262 & 0.272 & 0.544 \\
\hline W3L2 & 35.905 & 1500 & 0.260 & 0.293 & 0.518 \\
\hline W3L3 & 55.555 & 1200 & 0.254 & 0.302 & 0.553 \\
\hline W3L4 & 97.694 & 900 & 0.248 & 0.390 & 0.757 \\
\hline W3L5 & 159.361 & 700 & 0.243 & 1.042 & 0.949 \\
\hline
\end{tabular}

where $\eta$ is the viscosity of the helium, and $f$ is the frequency. We note that in writing the viscous term in Eq. (17), and in the following, we assume that the viscous penetration depth is small compared to the dimensions of the tuning fork.

The resonant frequency of the fork is inversely proportional to the square root of the effective mass, and hence the resonant frequency in liquid helium $f_{\mathrm{H}}$ may be found from

$$
\left(\frac{f_{0}}{f_{\mathrm{H}}}\right)^{2}=1+\frac{\beta V \rho_{\mathrm{H}}}{m_{\mathrm{eff}}}+\frac{B S}{m_{\mathrm{eff}}} \sqrt{\frac{\eta \rho_{\mathrm{nf}}}{\pi f_{0}}} .
$$

Viscous drag also leads to dissipation and hence contributes to the damping of the fork motion which can be written as ${ }^{8}$

$$
\Delta f_{2}^{V}=\frac{C S}{2 m_{\mathrm{eff}}} \sqrt{\frac{\rho_{\mathrm{nf}} \eta f_{0}}{\pi}}\left(\frac{f_{\mathrm{H}}}{f_{0}}\right)^{2},
$$

where $C$ is another geometrical factor of order of unity.

Measurements of the resonant frequencies and of the frequency widths of the fundamental resonances for various forks in liquid ${ }^{4} \mathrm{He}$ are shown in Fig. 4. Figure 4(a) shows the measured values of $\left(f_{0} / f_{\mathrm{H}}\right)^{2}$ plotted against the temperature. The solid lines show least-squares fits to the data using Eq. (19). The only fitting parameters are the two geometrical factors, $\beta$ and $B$, whose values are listed in Table II. Values for the helium density $\rho_{\mathrm{H}}$, the normal fraction $\rho_{\mathrm{nf}}$, and the viscosity $\eta$ were taken from Ref. 31 . Figure 4(b) shows the corresponding frequency widths of the resonances plotted against the temperature. The solid lines show least-squares fits to the hydrodynamic damping given by Eq. (20). The geometrical factor $C$ is the only fitting parameter in this case. The fitted values are again listed in Table II.

The quality of the fits indicates that the mass enhancement (frequency shift) is very well described by the hydrodynamic drag force at low frequencies. The fitting parameters are very similar for all of the lower frequency resonances, indicating that there is no significant dependence on the length of the fork prongs. However, for frequencies above $\sim 100 \mathrm{kHz}$, the data are not adequately described by hydrodynamic drag alone. This is clearly demonstrated by the poor fit to the data obtained with fork "W3L5" ( $\left.f_{0}=159 \mathrm{kHz}\right)$, shown in Fig. 4.

The resonant frequency of the first overtone mode of the longest fork, "W3S1," is sufficiently low that it should be described by hydrodynamic drag alone. So this fork provides an opportunity to test the hydrodynamic model for the first
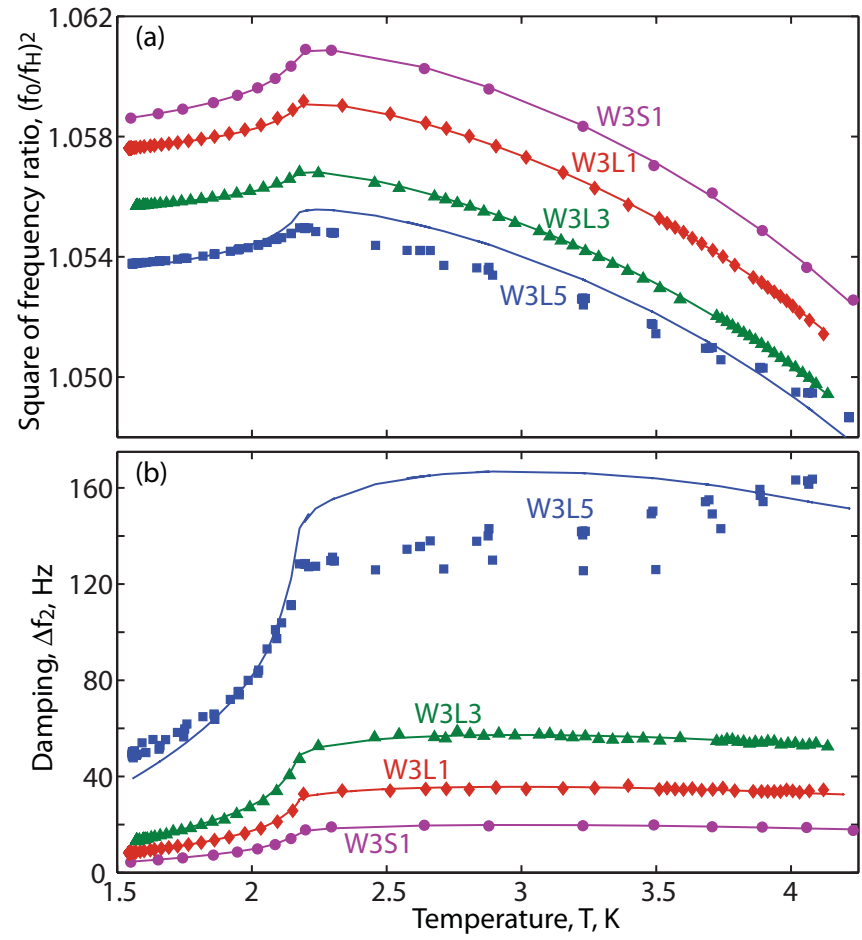

FIG. 4. (Color online) (a) The square of the frequency ratio (the resonant frequency in vacuum to that in liquid ${ }^{4} \mathrm{He}$ ) for the fundamental resonant mode of various tuning forks as a function of temperature. (b) The corresponding frequency widths of the resonances as a function of temperature. Solid lines give theoretical fits with fitting parameters listed in Table II (see text).

overtone mode. A comparison of the fundamental and first overtone modes for this fork is shown in Fig. 5. The solid lines show least-squares fits to the hydrodynamic equations. The quality of the fits is very good, which verifies that the hydrodynamic theory also works well for the first overtone mode. As shown in Table II, the fitting parameters are virtually identical for the two resonant modes. We infer that the geometrical factors $\beta, B$, and $C$ are mainly determined by the fork geometry perpendicular to the flow and are quite insensitive to the velocity profile along the length of the fork prongs.

\section{B. Acoustic drag}

The acoustic contribution to the damping of a tuning fork has been discussed previously, ${ }^{7,8,23}$ and theoretical models have been proposed to explain the measured frequency dependence of the acoustic drag. ${ }^{24}$ We consider two models to describe the dissipation due to sound emission, which we refer to as the "3D" model and the "2D" model. The details of the models are given in Ref. 24. Here we give the main results of the models and make a detailed comparison with our experimental results.

The tuning fork is modeled by four collinear point sources. In the 2D model, the sources emit cylindrical waves; the width of the prongs and the tip velocity amplitude govern the source strength to calculate the power emitted per unit length, which is then multiplied by an effective emitting length to give the total power dissipated. ${ }^{24}$ In the 3D model, the sources emit 

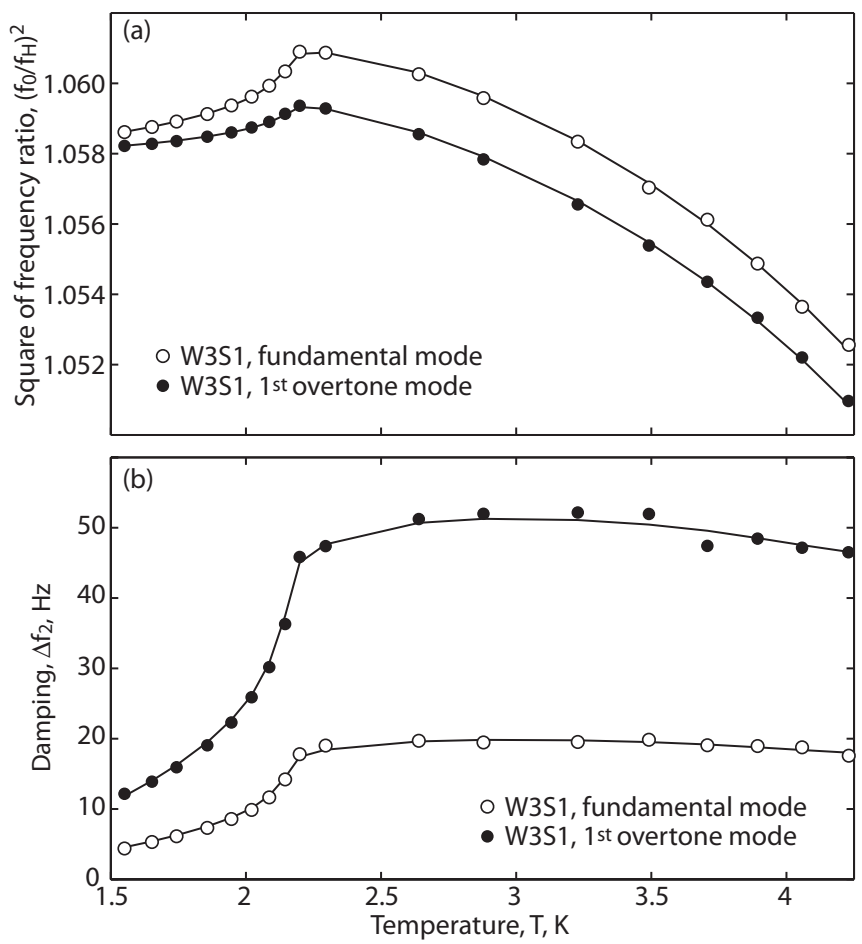

FIG. 5. (a) The square of the frequency ratio (the resonant frequency in vacuum to that in liquid ${ }^{4} \mathrm{He}$ ) for the longest fork "W3S1." (b) The corresponding frequency widths of the resonances as a function of temperature. Open and filled symbols correspond to the fundamental and the first overtone mode, respectively. Solid lines give theoretical fits to hydrodynamic drag with fitting parameters listed in Table II (see text).

spherical waves; the strength of the source is governed by the surface area of the prongs and the prong tip velocity. ${ }^{24} \mathrm{We}$ assume that our container (a long cylinder with a diameter of $\sim 5 \mathrm{~cm}$ ) can be considered to be infinite so that any acoustic energy emitted by the fork is carried away by the fluid. We see no evidence for acoustic standing waves being excited in our experiments, unlike what has been observed previously in closed volumes. ${ }^{23,24}$

The acoustic power dissipated by the tuning fork according to the two models are calculated in Ref. 24. The frequency width of the fork resonance is related to the power dissipation $\dot{Q}$ by

$$
\Delta f_{2}=\frac{\dot{Q}}{2 \pi m_{\mathrm{eff}}\left(v_{0}^{\mathrm{res}}\right)^{2}} .
$$

Using this expression and the results given in reference ${ }^{24}$, the acoustic contribution to the damping according to the $3 \mathrm{D}$ model is found to be ${ }^{33}$

$$
\begin{aligned}
\Delta f_{2}^{3 \mathrm{D}} & =C_{3 \mathrm{D}} \frac{\rho_{\mathrm{He}} W^{2} L_{e}^{2} f^{2}}{c m_{\mathrm{eff}}}\left(\frac{f_{\mathrm{H}}}{f_{0}}\right)^{2} \Sigma_{3 \mathrm{D}}, \\
\Sigma_{3 \mathrm{D}} & =\sum_{\substack{m=0 \\
\text { even }}}^{\infty}(2 m+1)\left[j_{m}\left(\frac{\pi f(2 T+D)}{c}\right)-j_{m}\left(\frac{\pi f D}{c}\right)\right]^{2},
\end{aligned}
$$

where $j_{m}()$ are spherical Bessel functions, $c$ is the first sound velocity in helium, $L_{e}=0.3915 L$ is the effective emitting length of a prong in the fundamental resonance mode, ${ }^{24}$ and $C_{3 \mathrm{D}}$ is a fitting parameter, expected to be of order unity.

For the case where the wavelength of the emitted sound is much larger than the relevant fork dimensions (the longwavelength limit), the expression given above can be further simplified by a Taylor expansion of the Bessel functions:

$$
\Delta f_{2}^{3 \mathrm{D}^{\prime}}=C_{3 \mathrm{D}^{\prime}} \frac{64 \pi^{4} 0.3915^{2}}{5} \frac{(T+D)^{2}}{\rho_{q}^{2}} \frac{\rho_{\mathrm{H}}}{c^{5}}\left(\frac{f_{\mathrm{H}}}{f_{0}}\right)^{2} m_{\mathrm{eff}} f_{\mathrm{H}}^{6} .
$$

We note that the ratio of resonant frequencies in helium and in vacuum is always close to unity. We also note that, since the effective mass is proportional to the prong length which varies as $f^{-0.5}$ according to $\mathrm{Eq}$. (2), the 3D model predicts that the acoustic drag varies as $f^{5.5}$.

Similarly, we find that the acoustic damping in the 2D model for sound emission can be expressed as

$$
\begin{gathered}
\Delta f_{2}^{2 \mathrm{D}}=C_{2 \mathrm{D}} \frac{\rho_{H e} W^{2} L_{e} f}{2 m_{\mathrm{eff}}}\left(\frac{f_{\mathrm{H}}}{f_{0}}\right)^{2} \Sigma_{2 \mathrm{D}} \\
\Sigma_{2 \mathrm{D}}=\sum_{\substack{m=-\infty \\
\text { even }}}^{\infty}\left[J_{m}\left(\frac{\pi f(2 T+D)}{c}\right)-J_{m}\left(\frac{\pi f D}{c}\right)\right]^{2},
\end{gathered}
$$

where $J_{m}()$ are cylindrical Bessel functions. ${ }^{24}$ In the longwavelength limit this simplifies to

$$
\Delta f_{2}^{2 \mathrm{D}^{\prime}}=C_{2 \mathrm{D}^{\prime}} \frac{3 \pi^{4} 0.3915 W T(T+D)^{2}}{\rho_{q}} \frac{\rho_{\mathrm{H}}}{c^{4}}\left(\frac{f_{\mathrm{H}}}{f_{0}}\right)^{2} f_{\mathrm{H}}^{5} .
$$

So in this case, the acoustic contribution to the damping is proportional to $f^{5}$, i.e., the $2 \mathrm{D}$ model has a marginally shallower frequency dependence compared to the 3D model in the long-wavelength limit.

Although the predictions of the long-wavelength limit are much clearer, it should be used with caution. The largest fork dimension is the prong length, $L$. For our longest fork, $L=3.5 \mathrm{~mm}$, while the emitted sound has a wavelength of $\sim 30$ $\mathrm{mm}$ for the fundamental mode and $\sim 4 \mathrm{~mm}$ for the first overtone mode. For the fork "W3L3" with a prong length of $1.2 \mathrm{~mm}$, the emitted sound wavelengths are $\sim 3 \mathrm{~mm}$ for the fundamental mode and $\sim 0.5 \mathrm{~mm}$ for the first overtone mode. So the long-wavelength limit may be a reasonable approximation for the fundamental mode, but it will not work well for the first overtone mode. A comparison of the three-dimensional model in its full form and in the long-wavelength approximation shows that the latter overestimates the contribution to the tuning fork width by $40 \%$ at $f_{0} \approx 340 \mathrm{kHz}$ and by $5 \%$ at $f_{0} \approx 100 \mathrm{kHz}$ for our tuning fork dimensions.

To compare the predictions of the acoustic models with our measurements we have subtracted the hydrodynamic contribution to the damping from the data shown in Fig. 3. The hydrodynamic contributions were obtained by fitting the width of the low-frequency resonances (below $60 \mathrm{kHz}$ ) 


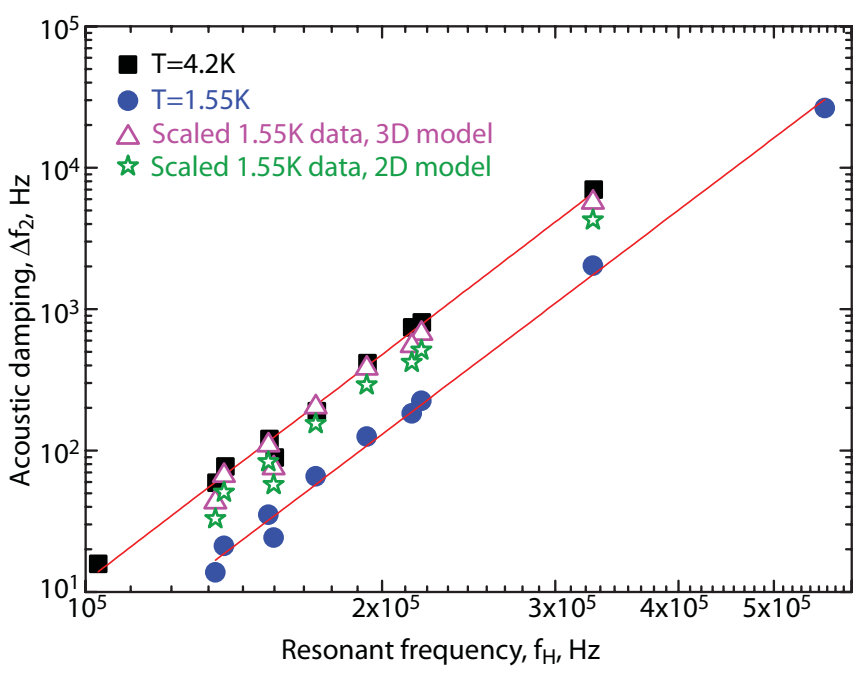

FIG. 6. (Color online) The acoustic damping of tuning forks as a function of frequency. The squares and circles correspond to the inferred acoustic contribution to the damping at 4.2 and $1.55 \mathrm{~K}$, respectively. Red lines are least-squares fits given by $\Delta f_{2}^{4.2 \mathrm{~K}}=2.7 \times$ $10^{-26} f^{5.33}$ and $\Delta f_{2}^{1.55 \mathrm{~K}}=1.6 \times 10^{-26} f^{5.26}$. Open triangles and stars show the $1.55 \mathrm{~K}$ data scaled using the full expressions for the $3 \mathrm{D}$ and 2D models, respectively (see text).

to a square-root frequency dependence. The fits for the two different temperatures shown were $\Delta f_{2}^{4.2 \mathrm{~K}}=0.22 f^{0.5}$ and $\Delta f_{2}^{1.55 \mathrm{~K}}=0.058 f^{0.5}$. We note that Eqs. (19) and (20) reproduce these fitted values using geometrical constants of $\beta=0.260, B=0.28$ and $C=0.542$, which agree well with averaged values given in Table II for the lower resonant frequencies.

Figure 6 shows the remaining acoustic contribution to the frequency width for resonance frequencies above $100 \mathrm{kHz}$, where acoustic emission is significant. Open squares and circles correspond to measurements at 4.2 and $1.55 \mathrm{~K}$ data, respectively. The solid lines in the figure show least-squares fits given by $\Delta f_{2}^{4.2 \mathrm{~K}}=2.7 \times 10^{-26} f^{5.33}$ and $\Delta f_{2}^{1.55 \mathrm{~K}}=1.6 \times$ $10^{-26} f^{5.26}$. Since the fitted power laws lie partway between the predictions of the $3 \mathrm{D}$ and $2 \mathrm{D}$ models in the long-wavelength limit, it is very difficult to distinguish them on this basis.

We can test the models further by investigating the ratio of the acoustic damping at $4.2 \mathrm{~K}$ to that at $1.55 \mathrm{~K}$. The predicted ratios for the two models are given by

$$
\begin{aligned}
& \frac{\Delta f_{2}^{3 \mathrm{D}}(4.2 \mathrm{~K})}{\Delta f_{2}^{3 \mathrm{D}}(1.55 \mathrm{~K})}=\frac{\rho_{4.2}}{\rho_{1.55}} \frac{c_{1.55}}{c_{4.2}} \frac{f_{4.2}^{4}}{f_{1.55}^{4}} \frac{\Sigma_{3 \mathrm{D}}(4.2 \mathrm{~K})}{\Sigma_{3 \mathrm{D}}(1.55 \mathrm{~K})}, \\
& \frac{\Delta f_{2}^{2 \mathrm{D}}(4.2 \mathrm{~K})}{\Delta f_{2}^{2 \mathrm{D}}(1.55 \mathrm{~K})}=\frac{\rho_{4.2}}{\rho_{1.55}} \frac{f_{4.2}}{f_{1.55}} \frac{\Sigma_{2 \mathrm{D}}(4.2 \mathrm{~K})}{\Sigma_{2 \mathrm{D}}(1.55 \mathrm{~K})},
\end{aligned}
$$

where $f_{\mathrm{T}}$ is the resonant frequency at temperature $T$. The values of the helium densities and of the first sound velocities are $\rho_{4.2}=125.41 \mathrm{~kg} \mathrm{~m}^{-3}, \rho_{1.55}=145.18 \mathrm{~kg} \mathrm{~m}^{-3}, c_{4.2}=$ $181.64 \mathrm{~m} \mathrm{~s}^{-1}$, and $c_{1.55}=235.05 \mathrm{~m} \mathrm{~s}^{-1} .31$

The triangles and stars in Fig. 6 show the acoustic damping for the $1.55 \mathrm{~K}$ data scaled to the expected damping at $4.2 \mathrm{~K}$ according to the 3D and 2D models as given by Eqs. (28) and (29), respectively. The scaling of the 3D model agrees

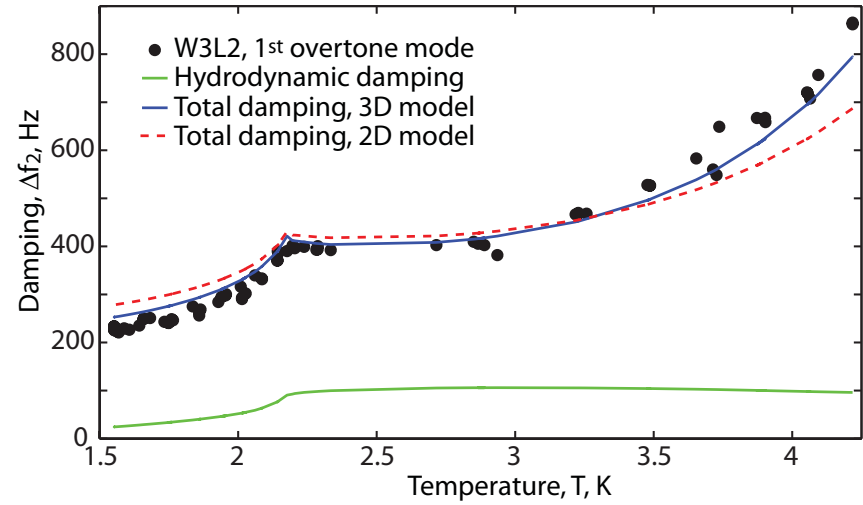

FIG. 7. (Color online) The frequency width of the first overtone resonance for the fork "W3L2" as a function of temperature. The solid green line shows the expected hydrodynamic contribution calculated using the coefficients for the fundamental mode. Red dashed and blue solid lines give the sums of the hydrodynamic and acoustic contributions using least-square fits to the acoustic damping expressions for the 2D and 3D models, respectively (see text).

very well with the data, whereas that of the $2 \mathrm{D}$ model lies significantly below the $4.2 \mathrm{~K}$ data.

Fits to the total damping at 4.2 and $1.55 \mathrm{~K}$, using the full expression for the 3D model, are shown in Fig. 3. The 3D model is found to agree very well with the data and the same fitting parameter, $C_{3 \mathrm{D}}=2.17$, is used for both temperatures.

The 3D model also agrees well with the measured temperature dependence of the acoustic damping. This is illustrated in Fig. 7, which shows the frequency width of the first overtone resonance for the fork "W3L2" as a function of temperature. The lower (green) line in the figure shows the hydrodynamic contribution to the damping, using Eq. (20) assuming the same geometrical factors used to fit the fundamental resonance properties as listed in Table II. The upper (blue) solid and (red) dashed lines give the sums of the hydrodynamic and acoustic contributions using the full expressions for the 3D and 2D models [Eqs. (22) and (25), respectively]. The fits have a single fitting parameter $C$ which governs the overall magnitude of the acoustic damping. The value of the fitting parameter for the different models and for three different fork resonances are listed in Table III. Although neither model reproduces the data perfectly, the $3 \mathrm{D}$ model gives a significantly better fit to the experiments.

\section{SUMMARY}

We have presented detailed measurements on quartz tuning forks over a wide frequency range, from 6.5 to $600 \mathrm{kHz}$,

TABLE III. The resulting fitting parameters obtained using leastsquares fits to the experimental data according to various acoustic emission models.

\begin{tabular}{lcccccc}
\hline \hline Fork & $\begin{array}{c}f_{0} \\
(\mathrm{kHz})\end{array}$ & $\begin{array}{c}L \\
(\mu \mathrm{m})\end{array}$ & $C_{3 \mathrm{D}}$ & $\begin{array}{c}C_{3 \mathrm{D}^{\prime}} \text { long } \\
\text { wavelength }\end{array}$ & $\begin{array}{c}C_{2 \mathrm{D}} \\
\text { wavelength }\end{array}$ \\
\hline W3L1fov & 139.383 & 1900 & 1.994 & 1.911 & 1.045 & 0.997 \\
W3L2fov & 220.948 & 1500 & 2.263 & 2.043 & 1.458 & 1.301 \\
W3L3fov & 338.990 & 1200 & 2.340 & 1.819 & 1.934 & 1.458 \\
\hline \hline
\end{tabular}


using the fundamental and first overtone resonance modes. The forks were all manufactured from a $75 \mu \mathrm{m}$ wafer, with nominally the same dimensions except for the length of the prongs. Several resonances were characterized at $4.2 \mathrm{~K}$ in vacuum. For the fundamental resonance modes, the fork calibration constants $a$ are found to be approximately one third of the theoretically expected values, in agreement with earlier measurements. Analytical calculations show that the effective mass of the tuning fork prongs is one quarter of the actual mass, independent of the resonance mode. This allows us to find experimental values for the fork calibration constants of the higher resonance modes and we present several measurements for the first overtone mode.

We have presented systematic measurements of drag forces on the tuning forks in normal and superfluid helium above $1.5 \mathrm{~K}$. At relatively low frequencies, the behavior is dominated by hydrodynamic drag associated with laminar normal fluid flow around the fork prongs. On increasing frequency, acoustic drag quickly dominates above $100 \mathrm{kHz}$. We have presented various measurements of the acoustic damping at high frequencies. We find that the 3D model of acoustic emission gives a good description of the experimental data.

Finally, we note that all of the measurements presented here were conducted at very low oscillation amplitudes so that the measured drag forces were proportional to the velocity. At much higher velocity amplitudes, quantum vortices are generated in superfluid helium and turbulent drag plays a significant role. The tuning fork arrays described here allow us to study vortex nucleation and turbulent drag over a broad frequency range. This will be reported elsewhere.

\section{ACKNOWLEDGMENTS}

We thank S. M. Holt, A. Stokes, and M. G. Ward for their excellent technical support, and P. Skyba for useful discussions. This research was supported by the UK EPSRC, by the European FP7 Programme MICROKELVIN, Project No. 228464, and by the research plans MS 0021620834 , GAČR 202 08/0276, of the Czech Republic.

\section{APPENDIX: EFFECTIVE MASS AND SPRING CONSTANT OF A RESONANT EULER BEAM}

With a harmonic drive uniformly distributed along the length of the beam, the equation of motion [Eq. (1)] can be written as

$$
\mu \frac{\partial^{2} u(x, t)}{\partial t^{2}}+\frac{\partial^{2}}{\partial x^{2}}\left(E I \frac{\partial^{2} u(x, t)}{\partial x^{2}}\right)=q_{0} e^{i \omega t} .
$$

Searching for solutions of the form $u(x, t)=X(x) e^{i \omega t}$ yields

$$
X(x)=-\frac{F}{\mu \omega^{2}}+c_{1} e^{b x}+c_{2} e^{-b x}+c_{3} e^{i b x}+c_{4} e^{-i b x},
$$

where $c_{1}, c_{2}, c_{3}$, and $c_{4}$ are integration constants and the wave number is given by

$$
b=\left(\frac{\mu \omega^{2}}{E I}\right)^{1 / 4}
$$

Substituting this in Eq. (A1) and applying the boundary conditions for fixed and free ends gives

$$
X(x)=\frac{q_{0}}{2 \omega^{2} \mu} \frac{g(x)}{[\cosh (b L) \cos (b L)+1]},
$$

where

$$
\begin{aligned}
g(x)= & \cos (b L) \cosh [b(L-x)]-\sinh (b L) \sin [b(L-x)] \\
& +\cosh (b L) \cos [b(L-x)]+\sin (b L) \sinh [b(L-x)] \\
& +\cos (b x)+\cosh (b x)-2 \cosh (b L) \cos (b L)-2 .
\end{aligned}
$$

Each resonant mode can be specified the integer $n$. The resonance condition can be written as

$$
\cosh \left(b_{n} L\right) \cos \left(b_{n} L\right)=-1 .
$$

Next, we evaluate the displacement profile along the beam for a given resonance mode, specified by the integer $n$. It is convenient to express this in terms of the amplitude of the free end, $X_{r}^{(n)}(x)=X^{(n)}(x) / X^{(n)}(x=L)$. Using Eq. (A6) we arrive at

$$
X_{r}^{(n)}(x)=\frac{1}{2} \frac{\sin \left(b_{n} L\right) \sinh \left(b_{n} L\right)\left[\cosh \left(b_{n} x\right)-\cos \left(b_{n} x\right)\right]-\left[\cos \left(b_{n} L\right) \sinh \left(b_{n} L\right)+\sin \left(b_{n} L\right) \cosh \left(b_{n} L\right)\right]\left[\sinh \left(b_{n} x\right)-\sin \left(b_{n} x\right)\right]}{\cosh \left(b_{n} L\right)+\cos \left(b_{n} L\right)} .
$$

The relative amplitudes can be used to express the effective mass and spring constants from Eqs. (5) and (6) as

$$
\begin{aligned}
m_{\mathrm{eff}}^{(n)} & =\mu \int_{0}^{L} X_{r}^{(n)}(x)^{2} d x \\
k_{n} & =E I \int_{0}^{L}\left[\frac{d^{2}\left(X_{r}^{(n)}(x)\right)}{d x^{2}}\right]^{2} d x .
\end{aligned}
$$

After evaluating the integrals, we find

$$
m_{\mathrm{eff}}^{(n)} \equiv m_{\mathrm{eff}}=\mu L / 4 ; \quad k_{n}=b_{n}^{4} E I L / 4 .
$$

These can be written in terms of the tuning fork dimensions using $\mu=\rho_{q} T W, I=W T^{3} / 12$, giving

$$
m_{\mathrm{eff}}=\rho_{q} T W L / 4 ; \quad k_{n}=b_{n}^{4} E W T^{3} L / 48 .
$$


It is useful to note that, substituting for $b_{n}$ from Eq. (2), the spring constants $k_{n}$ can be rewritten as

$$
k_{n}=\frac{1}{4} \mu \omega_{n}^{2} L=m_{\mathrm{eff}} \omega_{n}^{2},
$$

as one would expect for a harmonic oscillator.

*v.tsepelin@lancaster.ac.uk

${ }^{1}$ P. Günther, U. Ch. Fischer, and K. Dransfeld, Appl. Phys. B 48, 89 (1989).

${ }^{2}$ K. Karrai and R. D. Grober, Proc. SPIE 2535, 69 (1995).

${ }^{3}$ K. Karrai and R. D. Grober, Appl. Phys. Lett. 66, 1842 (1995).

${ }^{4}$ F. J. Giessibl, Appl. Phys. Lett. 73, 3956 (1998).

${ }^{5} \mathrm{~K}$. Karrai (unpublished), [http://www.nano.physik.uni-muenchen. de/publikationen/Preprints/p-00-03_Karrai.pdf].

${ }^{6}$ R. D. Grober, J. Acimovic, J. Schuck, D. Hessman, P. J. Kindlemann, J. Hespanha, S. Morse, K. Karrai, T. Tiemann, and S. Manus, Rev. Sci. Instrum. 71, 2776 (2000).

${ }^{7}$ D. O. Clubb, O. V. L. Buu, R. M. Bowley, R. Nyman, and J. R. Owers-Bradley, J. Low Temp. Phys 136, 1 (2004).

${ }^{8}$ R. Blaauwgeers, M. Blažková, M. Človečko, V. B. Eltsov, R. de Graaf, J. Hosio, M. Krusius, D. Schmoranzer, W. Schoepe, L. Skrbek, P. Skyba, R. E. Solntsev, and D. E. Zmeev, J. Low Temp. Phys. 146, 537 (2007).

${ }^{9}$ M. Blažková, M. Človečko, E. Gažo, L. Skrbek, and P. Skyba, J. Low Temp. Phys. 148, 305 (2007).

${ }^{10}$ M. Blažková, D. Schmoranzer, and L. Skrbek, Phys. Rev. E 75, 025302 (2007).

${ }^{11}$ M. Blažková, M. Človečko, V. B. Eltsov, E. Gazo, R. de Graaf, J. J. Hosio, M. Krusius, D. Schmoranzer, W. Schoepe, L. Skrbek, P. Skyba, R. E. Solntsev, and W. F. Vinen, J. Low Temp. Phys. 150, 525 (2008).

${ }^{12}$ G. A. Sheshin, A. A. Zadorozhko, E. Y. Rudavskii, V. K. Chagovets, L. Skrbek, and M. Blažková, Low Temp. Phys. 34, 875 (2008).

${ }^{13}$ M. Blažková, D. Schmoranzer, L. Skrbek, and W. F. Vinen, Phys. Rev. B 79, 054522 (2009).

${ }^{14}$ D. I. Bradley, M. J. Fear, S. N. Fisher, A. M. Guénault, R. P. Haley, C. R. Lawson, P. V. E. McClintock, G. R. Pickett, R. Schanen, V. Tsepelin, and L. A. Wheatland, J. Low Temp. Phys. 156, 116 (2009).

${ }^{15}$ D. Schmoranzer, M. Králová, V. Pilcová, W. F. Vinen, and L. Skrbek, Phys. Rev. E 81, 066316 (2010).

${ }^{16}$ M. Blažková, T. V. Chagovets, M. Rotter, D. Schmoranzer, and L. Skrbek, J. Low Temp. Phys. 150, 194 (2008).
${ }^{17}$ M. Blažková, D. Schmoranzer, and L. Skrbek, Low Temp. Phys. 34, 298 (2008).

${ }^{18}$ E. M. Pentti, J. T. Tuoriniemi, A. J. Salmela, and A. P. Sebedash, J. Low Temp. Phys. 150, 555 (2008).

${ }^{19}$ E. M. Pentti, J. T. Tuoriniemi, A. J. Salmela, and A. P. Sebedash, Phys. Rev. B 78, 064509 (2008).

${ }^{20}$ D. I. Bradley, M. Človečko, E. Gažo, and P. Skyba, J. Low Temp. Phys. 152, 147 (2008).

${ }^{21}$ D. I. Bradley, P. Crookston, S. N. Fisher, A. Ganshyn, A. M. Guénault, R. P. Haley, M. J. Jackson, G. R. Pickett, R. Schanen, and V. Tsepelin, J. Low Temp. Phys. , 157, 476 (2009).

${ }^{22}$ M. Človečko, E. Gažo, M. Kupka, M. Skyba, and P. Skyba, J. Low Temp. Phys. 162, 669 (2011).

${ }^{23}$ A. Salmela, J. Tuoriniemi, and J. Rysti, J. Low Temp. Phys. 162, 678 (2011).

${ }^{24}$ D. Schmoranzer, M. La Mantia, G. Sheshin, I. Gritsenko, A. Zadorozhko, M. Rotter, and L. Skrbek, J. Low Temp. Phys. 163, 317 (2011).

${ }^{25}$ The forks were manufactured by Statek Corporation, 512 N. Main Street, Orange, CA 92868, USA.

${ }^{26}$ J. M. Gere and S. P. Timoshenko, Mechanics of Materials, 3rd ed. (PWS-KENT Publishing Company, Boston, MA, 1990).

${ }^{27}$ S. P. Timoshenko, Philos. Mag. 41, 744 (1921).

${ }^{28}$ S. P. Timoshenko, Philos. Mag. 43, 125 (1922).

${ }^{29}$ D. I. Bradley, P. Crookston, M. J. Fear, S. N. Fisher, G. Foulds, D. Garg, A. M. Guénault, E. Guise, R. P. Haley, O. Kolosov, G. R. Pickett, R. Schanen, and V. Tsepelin, J. Low Temp. Phys. 161, 536 (2010).

${ }^{30}$ P. Skyba, J. Low Temp. Phys. 160, 219 (2010).

${ }^{31}$ R. J. Donnelly and C. F. Barenghi, J. Phys. Chem. Ref. Data 27, 1217 (1998).

${ }^{32}$ S. S. Chuang, Proceedings of the 35th Annual Frequency Control Symposium (USAERADCOM, Ft. Monmouth, NJ, 1981), p. 130.

${ }^{33}$ The expression for the power emission in Ref. 24 contains an error in the prefactor in front of the sum; the correct expression for the prefactor is $\rho_{q} \omega k W^{2} L_{e}^{2} U^{2} / 2 \pi$. Nevertheless, the numerical calculations presented in the paper, the fitting, and the longwavelength limit are correct. 